\title{
Videolaryngoscopy 2.0
}

\author{
J. Adam Law, MD (1) - George Kovacs, MD, MHPE
}

Received: 23 August 2021/Revised: 23 August 2021/Accepted: 23 August 2021/Published online: 16 December 2021

(C) Canadian Anesthesiologists' Society 2021

Videolaryngoscopy (VL) is great. For many reasons, it helps alleviate much of the stress associated with airway management. It's probably fair to say that, were there enough to go around (perhaps, if money was no object), we'd all like to use VL to facilitate every tracheal intubation. Indeed, published airway recommendations are beginning to suggest exactly this, resources allowing. ${ }^{1}$ This follows from the many studies and metaanalyses indicating that, compared with direct laryngoscopy (DL), VL is associated with increased tracheal intubation success and fewer complications. And many studies there are, with a PubMed search of the term "videolaryngoscopy" or "video laryngoscopy" currently yielding over 2900 results.

Retrospective cohort studies of large databases are a reasonable way to seek information about rare events, including the failure of airway management techniques such as DL and VL. While unable to prove causation, such studies often provide some interesting findings and can generate hypotheses regarding areas deserving of prospective evaluation. In this issue of the Journal, Jayaraj et al. report their findings from a retrospective review of tracheal intubation of a general surgical population using DL or VL in a large urban centre. ${ }^{2}$

\footnotetext{
J. A. Law, MD ( $ه)$

Department of Anesthesia, Pain Management and Perioperative Medicine, Dalhousie University, Halifax, NS, Canada

e-mail: jlaw@dal.ca

G. Kovacs, MD, MHPE

Department of Medical Neuroscience, Dalhousie University, Halifax, NS, Canada

Department of Emergency Medicine, Dalhousie University, Halifax, NS, Canada
}

Analyzing seven years (2011-2017) of data, they found a low incidence of difficult and failed tracheal intubation relative to standard figures, with a decreasing occurrence over time. The authors postulate that this may relate to increasing use of VL in primary and rescue capacities, which is not unreasonable. Other findings include good success rates of VL in rescuing unsuccessful first-attempt tracheal intubation facilitated by DL or VL.

Videolaryngoscopy has been with us for 20 years. Initially introduced with hyperangulated (acute angle) blades, video laryngoscopes are now available with a variety of blade shapes, including Macintosh geometry (Mac-VL). That apart, over these 20 years, there has not really been a single design feature or design objective that might define "second generation" VL, in contrast to the design modifications to supraglottic airways to help prevent aspiration of gastric contents. ${ }^{3}$ Nevertheless, as witnessed by the $>2900$ hits on VL in the PubMed search, there is a massive amount of published literature on the use of VL to facilitate tracheal intubation. So, absent a design feature that defines second generation VL, perhaps we can use the term "videolaryngoscopy 2.0" (VL 2.0) to draw attention to aspects of VL that we now know but that may not have been intuitive or evident when VL was first introduced. In this spirit, we offer the following observations.

\section{It's about the blade geometry, not the video}

Videolaryngoscopy 2.0 includes recognizing that most of the benefits of VL relate not to the video but to the laryngoscopy. The success of the laryngoscopy, in turn, relates to the shape of the blade on which the video chip is mounted. Some of the more battle-scarred among us may 
fondly remember the Bullard laryngoscope: now essentially a legacy device, it was a fibreoptic-based rigid laryngoscope with a $90^{\circ}$, hyperangulated blade. Very effective at delivering a full view of the larynx to a proximal eyepiece no matter how difficult the DL, there was nary a video chip in sight. Yet (at least for those able to master tube delivery), it was a formidable asset in the fight against the difficult airway. As with modern day hyperangulated blade VL, its strength lay in the capacity to slide the blade midline around the tongue, rather than to fight it, and to deliver the goods-usually a beautiful view of the larynx - to the viewing objective. Many contemporary hyperangulated VL blades feature somewhat less anterior blade angulation-often around 60-70 - but still enough to enable gratifying "around the corner" viewing for known or anticipated difficulty with DL and happily, with easier (but not always easy) tube delivery. Without doubt, having the larynx displayed larger-than-life and with a wide viewing angle on a video monitor during $\mathrm{VL}$ provides additional value over hunching over to squint through a small viewing objective, but ultimately, what's most important is seeing the larynx, however displayed.

Seeing the larynx of the difficult DL patient is less likely with Mac-VL. With Mac-VL, one is using traditional DL technique to compress and displace the tongue. While the view obtained on the video monitor is nice for all to see, the increment in laryngeal exposure over using traditional Macintosh DL when difficulty is anticipated or proven is usually less dramatic than when using a hyperangulated VL blade. Mac-VL is still wonderful, though, for its benefits in teaching DL to novices, to help deliver a view past a large moustache, lips, or teeth and to help engage others in the room with the airway management process. Without known or suspected adverse anatomy, Mac-VL is likely the best choice for the potentially soiled airway so that direct per-oral visualization can occur should the video camera become obscured, and yes, it may still provide a small increment in laryngeal exposure over traditional DL. Thus, VL 2.0 includes recognition that Mac-VL is probably best for more routine laryngoscopy when no great difficulty is anticipated, but that for known or suspected difficult DL, you need to reach for a videolaryngoscope with a hyperangulated blade.

\section{Referring accurately to VL}

Videolaryngoscopy 2.0 includes recognizing that the term "videolaryngoscopy" is passé without explicit follow-up reference to the exact device in question. There are simply too many devices now on the market with their differences in design, indications, and optimal techniques for use to get away with using the undifferentiated term. Equally, manufacturers now often market multiple different classes of videolaryngoscope under a single brand name. For example, while the original Karl Storz "C-MAC®" (Karl Storz SE \& Co. KG, Tuttlingen, Germany) referred only to a Macintosh-geometry bladed videolaryngoscope, there are now also C-MAC hyperangulated D-blades, C-MAC Miller VL blades (in pediatric sizes), a C-MAC flexible endoscope, and a C-MAC video-based stylet with deflectable tip. Indeed, on the company's website, Karl Storz refers to the C-MAC as a "system" 4 that supports multiple devices. Similar considerations apply to the GlideScope ${ }^{\circledR}$ (Verathon Inc., Bothell, WA, USA): previously synonymous with its original hyperangulated blade, there are now Macintosh and straight blade geometry videolaryngoscopes in the brand, together with a flexible endoscope. ${ }^{5}$ Thus, clinicians must document the exact VL blade they used, and authors must recognize that they can no longer simply state a brand name such as " $\mathrm{C}$ MAC" or "GlideScope" in their reports without stating the exact device in question. Peer reviewers and journal editors must also insist on this level of clarity. A study that reports impressive results with use of a "C-MAC" might appropriately be passed over by clinicians for translation into their practice if they are unsure of exactly which device is being referred to. Early reports on VL can be forgiven, when manufacturers had only a single VL device, but going forward, the free passes are no longer available. Videolaryngoscopy 2.0 includes use of precise terminology when describing VL.

\section{Knowing how to get the tube down}

Videolaryngoscopy 2.0 means knowing how to use the devices, based on which type of videolaryngoscope you're using. Clinicians facile in using both Macintosh and straight (e.g., Miller) blades for DL are no strangers to this concept - they differ with respect to blade insertion, managing the tongue and epiglottis, and shaping a styletted tracheal tube. Videolaryngoscopy is no different, in that there are differences in tracheal tube preparation and delivery between hyperangulated, Macintosh geometry, and channelled blade VL devices. Tube delivery with MacVL should not differ from Macintosh blade DL with or without adjunctive use of stylet or bougie; tracheal intubation using hyperangulated VL will be facilitated by not seeking a full view of the larynx, ${ }^{6}$ shaping a styleted tube similarly to the blade in use, and partial stylet withdrawal once the tip of the tube is just through the larynx. ${ }^{7}$ Tracheal tube delivery with hyperangulated VL blades might come more easily to those without many years of DL experience, as some aspects of optimal DL 
(e.g., striving for full laryngeal exposure) may be counterproductive when using hyperangulated VL, and engrained habits are hard to break. Nevertheless, regardless of clinician experience, VL 2.0 includes becoming knowledgeable about, and facile with, the subtleties of tube delivery with the different VL devices. ${ }^{8}$

\section{Limitations to VL}

Videolaryngoscopy 2.0 includes recognizing the limitations of videolaryngoscopes. While useful, tracheal intubation facilitated by VL does not have a $100 \%$ first attempt or overall success rate. As Jayaraj and colleagues have documented nicely, VL also doesn't have a $100 \%$ success rate used in a rescue capacity after failed DL or failed VL. ${ }^{2}$ In their retrospective study, after a failed first attempt at tracheal intubation in 91 patients using DL, VL (the only videolaryngoscope in use at their institution was the hyperangulated GlideScope GVL) was used for a second attempt in 52 patients, and succeeded in 41 (79\%). Perhaps even more interesting was their data for patients with failed first-attempt VL. Of 17 such patients, 11 (65\%) were successfully intubated on a second attempt with the same device, and 13 of the 17 patients, or $77 \%$, were eventually intubated using VL. While $77 \%$ is good, the clinician must be aware that tracheal intubation facilitated by hyperangulated VL can and does fail (causes of failure were not documented), when used in both primary and rescue capacities. Tellingly, there was no mention of moving on to DL after failed hyperangulated VL. This (or Mac-VL) has been reported to be effective in some such cases, ${ }^{9}, 10$ likely because of its straighter (and more familiar) pathway for tube passage. As Jayaraj et al. sagely point out in their discussion, use of the same VL device again after its first attempt failure could indicate fixation on a single technique, or possibly, not having thought about a Plan B when using what's perceived to be a very effective device. This could be concerning, and might warrant further study.

While VL is certainly useful, VL 2.0 includes recognizing that it is not a magic, one-size-fits-all bullet: the clinician must still have a plan for its potential failure. This may include a trial of DL or Mac-VL after the failure of hyperangulated VL, unless the former has already proven to have failed. In addition, as VL of any type will occasionally fail, the clinician is not absolved from careful, standard-of-care airway evaluation and planning on how most safely to proceed with airway management, including considering whether awake tracheal intubation is indicated. $^{7}$

\section{Systematic reviews and meta-analyses on VL vs DL}

Videolaryngoscopy 2.0 includes no longer combining studies of hyperangulated and Mac-VL into a single systematic review and meta-analysis. This has occurred in most such reviews of VL, ${ }^{11}$ many of which conclude that "VL" is superior to DL with respect to its primary outcome-often first attempt or overall tracheal intubation success. Interestingly, in reading beyond the abstract of these studies, when reported, subgroup analysis for some study outcomes differs between hyperangulated and MacVL blades. ${ }^{12}$ So here again, in trying to translate published findings into one's own practice, if both types of device are combined in a meta-analysis, to obtain the published outcome, which class of videolaryngoscope should one use? Future systematic reviews and meta-analyses simply must separate hyperangulated-VL from Mac-VL devices. Already, and certainly in the near future, there should be more than enough studies to allow this.

If anything, contemporary hyperangulated videolaryngoscopes might represent a second generation of the original fibreoptic-based hyperangulated devices like the Bullard laryngoscope. Both enable "around-thecorner" visualization. Nevertheless, the more important gift of the passage of time has been publication of a plethora of knowledge on both hyperangulated- and MacVL, meaning that we're way better informed on the sometimes overt, sometimes subtle aspects of VL use. Applying this knowledge-VL 2.0-will improve VL's utility to airway management and the practice of anesthesiology.

La vidéolaryngoscopie est extraordinaire. Pour de nombreuses raisons, elle aide à soulager une grande partie du stress associé à la prise en charge des voies aériennes. En fait, s'il y en avait assez (et si le budget était illimité), nous aimerions tous utiliser la vidéolaryngoscopie pour faciliter chaque intubation trachéale. Qui plus est, les recommandations publiées sur les voies aériennes commencent à suggérer précisément cela - lorsque les ressources le permettent. ${ }^{1}$ Ces recommandations découlent des nombreuses études et méta-analyses indiquant que, par rapport à la laryngoscopie directe, la vidéolaryngoscopie est associée à une augmentation du nombre d'intubations trachéales réussies et à une réduction des complications. Et il en existe, des études sur le sujet : une recherche PubMed des termes anglais «videolaryngoscopy» ou «video laryngoscopy » donne actuellement plus de 2800 résultats.

Les études de cohorte rétrospectives provenant des grandes bases de données sont une bonne façon de chercher des informations concernant des événements rares, y compris l'échec des techniques de prise en charge des 
voies aériennes telles que la laryngoscopie directe et la vidéolaryngoscopie. Bien qu'elles ne puissent pas prouver de lien de causalité, ces études fournissent bien souvent des résultats intéressants et peuvent générer des hypothèses concernant des domaines méritant une évaluation prospective. Dans ce numéro du Journal, Jayaraj et coll. rapportent leurs résultats d'un compte rendu rétrospectif de l'intubation trachéale d'une population chirurgicale générale par laryngoscopie directe et vidéolaryngoscopie dans un grand centre urbain. ${ }^{2}$ En analysant sept années (2011-2017) de données, ces auteurs ont constaté une faible incidence d'intubation trachéale difficile et ratée par rapport aux chiffres standard, avec une occurrence décroissante au fil du temps. Les auteurs postulent que cela pourrait être lié à l'utilisation croissante de la vidéolaryngoscopie tant dans un rôle primaire que de sauvetage, ce qui n'est pas déraisonnable. D'autres résultats citent aussi les bons taux de réussite de la vidéolaryngoscopie pour reprendre une première tentative d'intubation trachéale infructueuse par laryngoscopie directe et vidéolaryngoscopie.

La vidéolaryngoscopie est utilisée depuis 20 ans. Elle a été initialement introduite avec des lames hyperangulées (angle aigu), qui sont maintenant disponibles auprès de plusieurs fabricants dans une variété de formes, y compris les lames de forme Macintosh adaptées pour les vidéolaryngoscopes (Mac-VL). Mis à part cette nouveauté, au cours des vingt années, aucune caractéristique de conception ou objectif de conception unique ne pourrait nous permettre d'identifier une soidisant «deuxième génération » de vidéolaryngoscopes, contrairement aux modifications de conception des dispositifs supraglottiques visant à prévenir l'aspiration du contenu gastrique. ${ }^{3}$ Néanmoins, comme en témoignent les plus de 2800 résultats touchant à la vidéolaryngoscopie dans notre recherche PubMed, il existe une quantité phénoménale de littérature publiée sur l'utilisation de la vidéolaryngoscopie pour faciliter l'intubation trachéale. Donc, en l'absence d'un trait de conception qui nous permettrait de définir la vidéolaryngoscopie de deuxième génération, peut-être pouvons-nous utiliser le terme de « vidéolaryngoscopie 2.0 » pour attirer l'attention sur les aspects de la vidéolaryngoscopie que nous connaissons maintenant, mais qui n'étaient peut-être pas intuitifs ou évidents lorsque cette modalité a été introduite pour la première fois. C'est dans cet esprit que nous vous soumettons les observations suivantes.

\section{Tout est dans la géométrie de la lame, pas dans la vidéo}

La vidéolaryngoscopie 2.0 comporte le fait de reconnaître que la plupart des avantages de la vidéolaryngoscopie ne touchent pas vraiment à la vidéo, mais plutôt à la laryngoscopie. La réussite de la laryngoscopie, à son tour, est liée à la forme de la lame sur laquelle la caméra est montée. Les plus aguerris d'entre nous se souviennent peut-être avec nostalgie du laryngoscope Bullard: aujourd'hui essentiellement un dispositif patrimonial, il s'agissait d'un laryngoscope rigide à base de fibre optique avec une lame hyperangulée à $90^{\circ}$, très efficace pour fournir une vue complète du larynx à un oculaire proximal et ce, quelle que soit la difficulté de la laryngoscopie directe - et pourtant, il ne disposait d'aucune caméra. Malgré tout, (du moins pour ceux capables de maîtriser le bon positionnement du tube), il s'agissait d'un atout incontestable dans notre lutte contre les voies aériennes difficiles. Tout comme avec la lame hyperangulée du vidéolaryngoscope d'aujourd'hui, la force de ce dispositif résidait dans la possibilité, pour l'opérateur, de faire glisser la lame à mi-chemin autour de la langue, plutôt que d'aller contre cette dernière, et de fournir une vue magnifique du larynx comme objectif de visualisation. De nombreuses lames de vidéolaryngoscopes hyperangulées contemporaines présentent une angulation de lame un peu moins antérieure - souvent autour de $60-70^{\circ}$ - mais suffisante pour permettre une visualisation gratifiante pour déterminer s'il y aura des difficultés connues ou anticipées avec la laryngoscopie directe et heureusement, avec un positionnement du tube plus aisé (même si pas toujours facile). Il ne fait aucun doute qu'une vue plus grande que nature du larynx, avec un grand angle de vision, affichée sur un moniteur vidéo pendant la vidéolaryngoscopie, offre une valeur ajoutée par rapport au fait de se pencher pour s'user les yeux à regarder à travers un petit oculaire; mais en fin de compte, ce qui est le plus important est de voir le larynx, quelle que soit l'image.

Il est moins évident, avec une lame Mac pour vidéolaryngoscopie, de voir le larynx d'un patient chez qui la laryngoscopie directe est difficile. Avec ce type de dispositif, on utilise une technique de laryngoscopie directe traditionnelle pour comprimer et déplacer la langue. Bien qu'il soit agréable que tous puissent admirer la vue obtenue sur le moniteur vidéo, l'augmentation de l'exposition laryngée par rapport à l'utilisation d'une laryngoscopie directe avec Macintosh traditionnelle, lorsque des difficultés sont anticipées ou avérées, est généralement moins dramatique que lors de l'utilisation d'une lame de vidéolaryngoscope hyperangulée. La vidéolaryngoscopie Mac demeure cependant merveilleuse, notamment pour ses avantages en matière d'enseignement de la laryngoscopie directe aux novices, en raison de sa capacité à offrir une vue malgré une belle moustache touffue, des lèvres ou des dents imposantes, et pour aider à impliquer les autres intervenants dans la pièce dans le processus de prise en charge des voies aériennes. En l'absence d'une anatomie 
difficile connue ou soupçonnée, la vidéolaryngoscopie Mac est probablement le meilleur choix pour des voies aériennes potentiellement souillées, de sorte qu'une visualisation directe par voie orale puisse être obtenue même si la caméra vidéo devient obscurcie, et oui, elle pourrait encore offrir une légère augmentation de l'exposition laryngée par rapport à une laryngoscopie directe traditionnelle. Ainsi, la vidéolaryngoscopie 2.0 comporte le fait d'être conscient que la vidéolaryngoscopie Mac demeure probablement le meilleur choix pour une laryngoscopie de routine lorsqu'aucune difficulté majeure n'est anticipée, mais que, pour une laryngoscopie directe difficile connue ou soupçonnée, il est plus judicieux d'utiliser un vidéolaryngoscope avec une lame hyperangulée.

\section{Parler avec précision de la vidéolaryngoscopie}

La vidéolaryngoscopie 2.0 comprend la reconnaissance que le terme «vidéolaryngoscopie » est dépassé s'il ne fait pas explicitement référence à un dispositif en particulier. À l'heure actuelle, il existe simplement trop de dispositifs sur le marché avec des différences de conception, d'indications et de techniques optimales pour leur utilisation pour s'en tirer en utilisant ce terme générique. De la même manière, les fabricants commercialisent maintenant souvent plusieurs classes différentes de vidéolaryngoscopes avec un seul nom de marque. Par exemple, alors que le «CMAC ${ }^{\circledR}$ » original de Karl Storz (Karl Storz SE \& Co. KG, Tuttlingen, Allemagne) faisait uniquement référence à un vidéolaryngoscope à lame Macintosh, il existe aujourd'hui des lames hyperangulées C-MAC pour la laryngoscopie directe, des lames C-MAC Miller pour la vidéolaryngoscopie (dans les tailles pédiatriques), un endoscope flexible C-MAC et un stylet vidéo C-MAC avec pointe articulée. En effet, sur le site web de l'entreprise, Karl Storz qualifie le C-MAC de «système ${ }^{4}$ qui prend en charge plusieurs dispositifs. Des considérations similaires s'appliquent au GlideScope $\AA$ (Verathon Inc., Bothell, WA, États-Unis) : auparavant synonyme de sa lame hyperangulée originale, la marque offre aujourd'hui des vidéolaryngoscopes à lame Macintosh et à lame droite, ainsi qu'un endoscope flexible. ${ }^{5}$ Ainsi, les cliniciens doivent documenter la lame de vidéolaryngoscopie exacte qu'ils ont utilisée, et les auteurs doivent être conscients qu'ils ne peuvent plus simplement indiquer un nom de marque tel que « $\mathrm{C}$ $\mathrm{MAC} » \mathrm{ou}$ « GlideScope » dans leurs comptes rendus, sans indiquer le dispositif exact en question. Les réviseurs pairs et les rédacteurs de revues doivent également insister sur ce niveau de précision. Une étude rapportant des résultats impressionnants avec l'utilisation d'un «C-MAC » pourrait être tout simplement - et justement - ignorée par un clinicien qui déciderait de ne pas l'appliquer dans sa pratique s'il n'est pas sûr de savoir exactement de quel dispositif il est question. On peut certes pardonner aux premiers comptes rendus sur la vidéolaryngoscopie leur manque de précision, étant donné que les fabricants n'offraient alors qu'un seul dispositif de vidéolaryngoscopie; en revanche, à l'avenir, cela n'est plus acceptable. La vidéolaryngoscopie 2.0 englobe l'utilisation d'une terminologie précise lors de la description du vidéolaryngoscope.

\section{Savoir comment intuber}

La vidéolaryngoscopie 2.0 signifie savoir comment utiliser les dispositifs, en fonction du type de vidéolaryngoscope que vous utilisez. Les cliniciens à l'aise avec les lames Macintosh et les lames droites (par exemple, des lames Miller) pour la laryngoscopie directe connaissent bien ce concept - elles diffèrent en ce qui concerne l'insertion de la lame, la gestion de la langue et de l'épiglotte et la mise en forme d'un tube trachéal avec stylet. C'est la même chose pour la vidéolaryngoscopie : il existe des différences dans la préparation et le positionnement des tubes trachéaux entre les dispositifs hyperangulés, ceux avec des lames Macintosh, et les dispositifs de vidéolaryngoscopie à lame canalisée. Le positionnement du tube avec un vidéolaryngoscope Mac ne devrait pas différer d'une laryngoscopie directe avec une lame Macintosh avec ou sans utilisation d'appoint de stylet ou de bougie; l'intubation trachéale à l'aide d'un vidéolaryngoscope hyperangulé sera plus facile si l'on ne cherche pas à obtenir une vue complète du larynx, ${ }^{6}$ si l'on façonne un tube avec un stylet de la même forme que la lame utilisée, et si l'on retire partiellement le stylet dès que l'extrémité du tube a passé le larynx. ${ }^{7}$ Le positionnement d'un tube trachéal avec une lame de vidéolaryngoscope hyperangulée pourrait être plus facile pour ceux qui ne pratiquent pas la laryngoscopie directe depuis de nombreuses années, car certains aspects d'une laryngoscopie directe optimale (par exemple, la recherche d'une exposition complète du larynx) pourraient être contre-productifs lors de l'utilisation d'un vidéolaryngoscope hyperangulé, et il est particulièrement difficile de se défaire de nos habitudes bien enracinées. Néanmoins, quelle que soit l'expérience du clinicien, la vidéolaryngoscopie 2.0 implique de se familiariser avec les subtilités de positionnement de tubes avec différents dispositifs de vidéolaryngoscopie - et de les maîtriser. ${ }^{8}$ 


\section{Limitations de la vidéolaryngoscopie}

La vidéolaryngoscopie 2.0 implique de reconnaître les limites des vidéolaryngoscopes. Bien qu'utile, l'intubation trachéale facilitée par vidéolaryngoscopie n'a pas un taux de réussite global de $100 \%$ à la première tentative. Comme Jayaraj et ses collègues l'ont bien documenté, la vidéolaryngoscopie n'a pas non plus un taux de réussite de $100 \%$ lorsqu'elle est utilisée comme modalité de sauvetage après un échec de laryngoscopie directe ou de vidéolaryngoscopie. ${ }^{2}$ Dans leur étude rétrospective, après une première tentative infructueuse d'intubation trachéale chez 91 patients utilisant la laryngoscopie directe, la vidéolaryngoscopie (le seul vidéolaryngoscope utilisé dans leur établissement était le GlideScope GVL hyperangulé) a été utilisée pour la deuxième tentative chez 52 patients, et a réussi chez $41(79 \%)$. Leurs données concernant les patients chez qui la première tentative de vidéolaryngoscopie avait échoué sont peut-être encore plus intéressantes : parmi ces 17 patients, 11 (65\%) ont été intubés avec succès lors d'une deuxième tentative avec le même dispositif, et 13 des 17 patients, soit $77 \%$, ont finalement pu être intubés à l'aide du vidéolaryngoscope. Bien que $77 \%$ soit un bon pourcentage, le clinicien doit être conscient que l'intubation trachéale facilitée par un vidéolaryngoscope hyperangulé peut échouer et échoue (les causes d'échec n'étaient pas documentées) lorsqu'un tel dispositif est utilisé en modalité d'intubation principale et de sauvetage. Fait révélateur, il n'est jamais fait mention d'un passage à la laryngoscopie directe après un échec avec un vidéolaryngoscope hyperangulé. Il a été rapporté que cette technique (ou la vidéolaryngoscopie avec lame Mac) pouvait être efficace dans de tels cas, ${ }^{9},{ }^{10}$ probablement en raison du chemin plus droit (et plus familier) pour le passage du tube. Comme Jayaraj et coll. le mentionnent avec sagesse dans leur discussion, l'utilisation d'un même dispositif de vidéolaryngoscopie après une première tentative infructueuse pourrait indiquer une fixation de l'opérateur sur une seule technique, ou peut-être, le fait de ne pas avoir pensé à un plan B lorsque l'opérateur utilise ce qu'il considère comme un appareil très efficace. Cela pourrait être inquiétant et justifier une étude plus approfondie.

Bien que la vidéolaryngoscopie soit certainement utile, la vidéolaryngoscopie 2.0 implique la reconnaissance qu'il ne s'agit pas d'une solution miraculeuse et unique : le clinicien doit toujours disposer d'un plan B en cas d'échec potentiel. Un tel plan peut inclure une tentative de laryngoscopie directe ou de vidéolaryngoscopie avec lame Mac après un échec d'intubation à l'aide d'un vidéolaryngoscope hyperangulé, à moins que ces techniques ne se soient déjà avérées inefficaces. De plus, comme la vidéolaryngoscopie, de quelque type que ce soit, peut occasionnellement échouer, le clinicien a le devoir de procéder à une évaluation minutieuse et selon la norme de soins des voies aériennes et de planifier la façon la plus sécuritaire de prendre en charge les voies aériennes de son patient, notamment d'évaluer si une intubation trachéale éveillée serait indiquée. ${ }^{7}$

\section{Revues systématiques et méta-analyses comparant vidéolaryngoscopie et laryngoscopie directe}

La vidéolaryngoscopie 2.0 implique de ne plus combiner les études sur les vidéolaryngoscopes hyperangulés et ceux équipés d'une lame Mac dans une seule revue systématique et méta-analyse. La plupart des revues sur la vidéolaryngoscopie le font, ${ }^{11}$ et bon nombre concluent que la « vidéolaryngoscopie » est supérieure à la laryngoscopie directe en ce qui concerne leur critère d'évaluation principal - souvent la réussite de l'intubation trachéale à la première tentative ou globale. Fait intéressant, si l'on s'attarde à lire davantage que le seul résumé de ces études, lorsqu'ils sont rapportés, les résultats de certaines études diffèrent lorsqu'il est question de lames hyperangulées et de lames Mac. ${ }^{12}$ Donc, là encore, si l'on tente de traduire les résultats publiés dans notre propre pratique, si les deux types de dispositifs sont combinés dans une méta-analyse, pour obtenir le résultat publié, quelle classe de vidéolaryngoscope faut-il utiliser? À l'avenir, les revues systématiques et méta-analyses devront simplement distinguer les appareils de vidéolaryngoscopie hyperangulés des appareils avec lames Mac. Déjà, et certainement dans un avenir proche, il devrait y avoir plus qu'assez d'études pour permettre cette distinction.

En effet, les vidéolaryngoscopes hyperangulés actuels pourraient représenter une deuxième génération des dispositifs hyperangulés originaux à base de fibres optiques tels que le laryngoscope Bullard. Les deux types permettent une visualisation "passé l'angle ». Néanmoins, ce que le passage du temps a pu nous offrir de mieux, c'est la publication d'une pléthore de connaissances tant sur les vidéolaryngoscopes hyperangulés que sur ceux munis de lames Mac : nous sommes donc bien mieux informés quant aux aspects parfois manifestes, parfois subtils de l'utilisation de la vidéolaryngoscopie. En appliquant ces connaissances, la vidéolaryngoscopie 2.0 améliorera l'utilité de la vidéolaryngoscopie pour la prise en charge des voies aériennes et la pratique de l'anesthésiologie.

\section{Disclosures None.}

\section{Funding statement None.}


Editorial responsibility This submission was handled by Dr. Philip M. Jones, Deputy Editor-in-Chief, Canadian Journal of Anesthesia.

Déclaration Aucune.

Déclaration de financement Aucune.

Responsabilité éditoriale Cet article a été traité par Dr Philip M. Jones, rédacteur en chef adjoint, Journal canadien d'anesthésie.

\section{References}

1. Law JA, Duggan LV, Asselin M, et al. Canadian Airway Focus Group updated consensus-based recommendations for management of the difficult airway: part 1. Difficult airway management encountered in an unconscious patient. Can $\mathbf{J}$ Anesth 2021; 68: 1373-1404. DOI: https://doi.org/10.1007/ s12630-021-02007-0.

2. Jayaraj AK, Siddiqui N, Abdelghany SM, Balki M. Management of difficult and failed intubation in the general surgical population-a historical cohort study in a tertiary care centre. Can J Anesth 2022; DOI: https://doi.org/10.1007/s12630-02102161-5.

3. Cook TM. Third generation supraglottic airway devices: an undefined concept and misused term. Time for an updated classification of supraglottic airway devices. Br J Anaesth 2015; 115: 633-4.

4. Karl Storz - Endoskope. The C-MAC(R) System. Available from URL: www.karlstorz.com/cps/rde/xbcr/karlstorz_assets/ASSETS/ 2136550.pdf (accessed August 2021).

5. Verathon. GlideScope Spectrum Single-Use Laryngoscope. Available from URL: https://www.verathon.com/glidescopespectrum-single-use/ (accessed August 2021).
6. Gu Y, Robert J, Kovacs $G$, et al. A deliberately restricted laryngeal view with the GlideScope $(\mathrm{R})$ video laryngoscope is associated with faster and easier tracheal intubation when compared with a full glottic view: a randomized clinical trial. Can J Anesth 2016; 63: 928-37.

7. Law JA, Duggan LV, Asselin M, et al. Canadian Airway Focus Group updated consensus-based recommendations for management of the difficult airway: part 2. Planning and implementing safe management of the patient with an anticipated difficult airway. Can J Anesth 2021; 68: 1405-36. DOI: https://doi.org/10.1007/s12630-021-02008-z.

8. Kovacs $G$, Levitan $R$. Redirecting the laryngoscopy debate and optimizing emergency airway management. Acad Emerg Med 2020; 27: 1366-9.

9. Aziz, MF, Healy D, Kheterpal S, Fu RF, Dillman D, Brambrink $A M$. Routine clinical practice effectiveness of the Glidescope in difficult airway management: an analysis of 2,004 Glidescope intubations, complications, and failures from two institutions. Anesthesiology 2011; 114: 34-41.

10. Aziz MF, Abrons RO, Cattano D, et al. First-attempt intubation success of video laryngoscopy in patients with anticipated difficult direct laryngoscopy: a multicenter randomized controlled trial comparing the C-MAC D-blade versus the GlideScope in a mixed provider and diverse patient population. Anesth Analg 2016; 122: 740-50.

11. Downey AW, Duggan LV, Law JA. A systematic review of metaanalyses comparing direct laryngoscopy and videolaryngscopy. Can J Anesth 2021; 68: 706-14 DOI: https://doi.org/10.1007/ s12630-021-01921-7.

12. Lewis SR, Butler AR, Parker J, Cook TM, Smith AF. Videolaryngoscopy versus direct laryngoscopy for adult patients requiring tracheal intubation. Cochrane Database Syst Rev 2016; DOI: https://doi.org/10.1002/14651858.CD011136.pub2.

Publisher's Note Springer Nature remains neutral with regard to jurisdictional claims in published maps and institutional affiliations. 